\title{
Design and development with educational purposes of an Optical Spectrum Analyzer for the visible range and of an Optical Time Domain Reflectometer for the second window
}

G. Durana, G. Aldabaldetreku, J. Zubia, M. A. Illarramendi, A. Berganza, et al.

G. Durana, G. Aldabaldetreku, J. Zubia, M. A. Illarramendi, A. Berganza, I. Bikandi, "Design and development with educational purposes of an Optical Spectrum Analyzer for the visible range and of an Optical Time Domain Reflectometer for the second window," Proc. SPIE 9666, 11th Education and Training in Optics and Photonics Conference, 96661T (5 June 2009); doi: $10.1117 / 12.2208088$ 


\title{
Design and development with educational purposes of an Optical Spectrum Analyzer for the visible range and of an Optical Time Domain Reflectometer for the second window
}

\author{
G. Durana, ${ }^{1}$ G. Aldabaldetreku, ${ }^{1}$ J. Zubia, ${ }^{1}$ M. A. Illarramendi, ${ }^{2}$ A. Berganza, ${ }^{1}$ \\ and I. Bikandi ${ }^{1}$ \\ ${ }^{1}$ Departamento de Electrónica y Telecomunicaciones, ETSI de Bilbao. University of the \\ Basque Country (UPV/EHU). Alda Urquijo s/n, E-48013 Bilbao (Spain) \\ ${ }^{2}$ Departamento de Física Aplicada I, ETSI de Bilbao. University of the Basque Country \\ (UPV/EHU). Alda Urquijo s/n, E-48013 Bilbao (Spain)
}

\begin{abstract}
We present in this paper the detailed design, development, and implementation of two optical measurement systems that serve as teaching devices for undergraduate students. The building of both prototypes has been carried out by several students for their undergraduate thesis project. Both systems are highly modular and each of their functional blocks are clearly separated and labeled so that students can immediately identify their constituent parts. In both cases, the hardware consists of optoelectronic devices and mechanical parts that are fully automated and controlled by the corresponding Windows application developed ad hoc. Our Optical Spectrum Analyzer is, as far as we know, the first system designed for highly multimode polymer optical fibers operating in the visible region. As for the Optical Time Domain Reflectometer, it is not only suitable for educational experimental measurements, but it can also be compared with commercial systems, since it works in the second transmission window.
\end{abstract}

Keyword list: Fiber optics; Fiber measurements; Fiber optics and optical communications.

\section{INTRODUCTION}

Optical Spectrum Analyzers (OSAs) and Optical Time Domain Reflectometers (OTDRs) are two optical measurement systems widely used by the scientific community as well as in telecom applications. The former separate the optical signal into its constituent wavelengths, allowing the measurement of the spectral profile of the signal as a function of the wavelength, whereas the latter measure the power loss with the distance within optical fibers, enabling the detection of faults, splices, and bends in optical links. ${ }^{1}$

A wide variety of commercial solutions is currently available from different manufacturers, ranging from modest equipments to extremely sophisticate devices. Nevertheless, the compact designs exhibited by most of these commercial solutions are not suitable from a didactic point of view: they do not serve to show undergraduate students the underlying functional blocks that made up each measurement system and the relationships between them.

For this reason, we have coordinated the design and development of two didactic prototypes (an OSA and an OTDR), with their functional blocks clearly separated and labeled. Being the size and complexity of this problem appropriate enough for undergraduate students, we have entrusted several students with the task of implementing and building both prototypes for their undergraduate thesis project.

In this paper, we will explain, concisely, the structure of both prototypes, the specifications of the main components that constitute each prototype, and how these components are interrelated. These explanations will be supported by several block diagrams and photographs.

\section{OPTICAL SPECTRUM ANALYZER}

One of the most remarkable features of our OSA prototype is that it has been specifically designed to handle optical signals obtained from highly multimode polymer optical fibers operating in the visible region and in part 
of the near-infrared region $(400-850 \mathrm{~nm})$. This OSA prototype consists of three main components, as shown in Fig. 1.

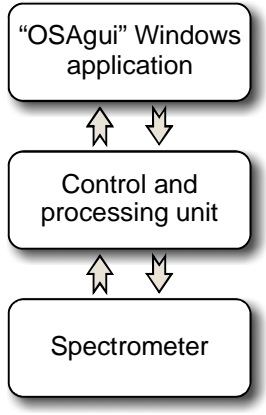

(a) Block diagram.

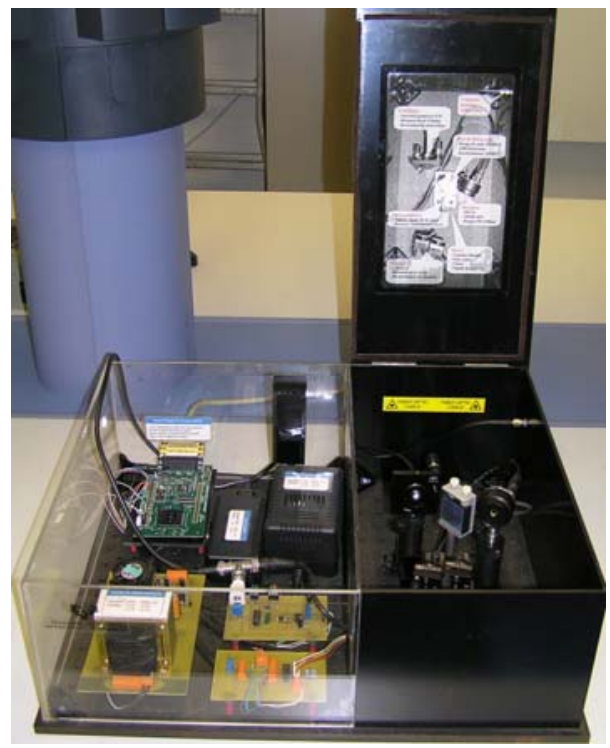

(b) Photograph.

Figure 1. Overall block diagram and photograph of the OSA prototype.

- Spectrometer: the optical part that separates the optical signal into its constituent wavelengths and converts it to an electrical signal.

- Control and processing unit: a general purpose Digital Signal Processor (DSP) with custom-made electrical circuits that controls the spectrometer and processes the electrical signal obtained from the latter.

- "OSAgui" Windows application: a graphical user interface that manages the DSP and displays the measurement results.

\subsection{Spectrometer}

The main purpose of the spectrometer is to separate the optical signal into its constituent wavelengths. Figure 2 shows the design and final implementation of the spectrometer.

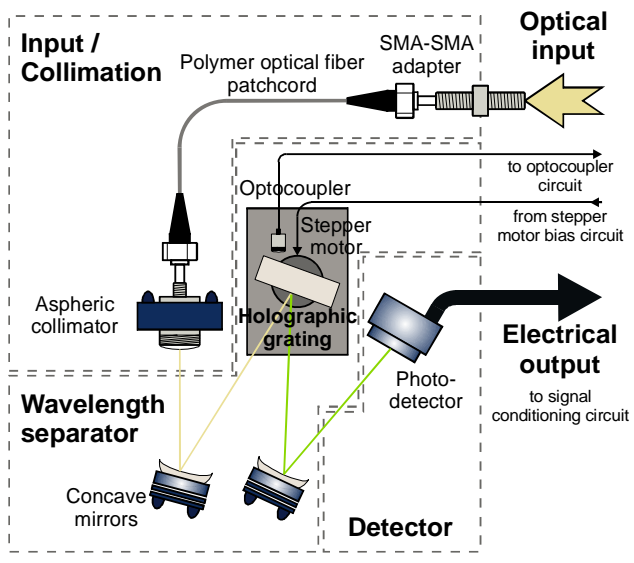

(a) Block diagram.

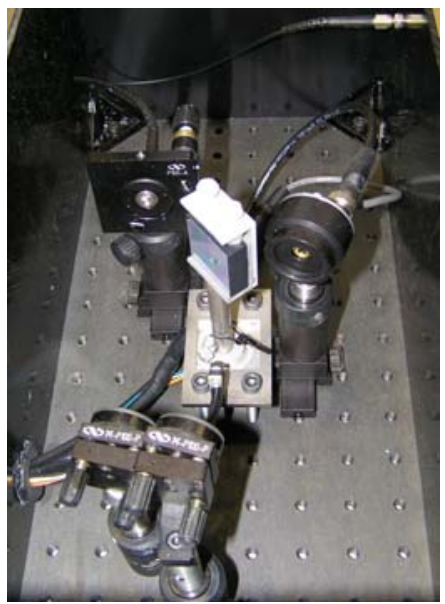

(b) Photograph.

Figure 2. Block diagram and photograph of the spectrometer. 
The main components of the spectrometer can also be separated into three main blocks:

- Input/Collimation: it leads the incoming optical signal to a Thorlabs F230SMA-A aspheric collimator. ${ }^{2}$ This aspheric lens ensures that the optical signal impinging on the following block consists only of parallel beams regardless of the light launching conditions at the optical input.

- Wavelength separator: the incoming optical signal from the aspheric collimator impinges on a Newport 10D20ER.1 concave mirror ${ }^{3}$ and then it is directed to a reflective holographic grating. This holographic grating, a NT43-225 model from Edmund Optics ${ }^{4}$ with a resolution of 2400 grooves per millimeter and suitable for the visible region, is the core component of the spectrometer: it separates the incident light into its constituent wavelength components, being each wavelength reflected into a different direction according to the grating equation ${ }^{5}$

$$
a\left(\sin \theta_{m}-\sin \theta_{i}\right)=m \lambda,
$$

$a$ being the spacing of the grooves (i.e., $a=1 / 2400 \mathrm{~mm}$ ), $\theta_{i}$ the angle of incidence, $m$ the order number ( $m=1$ in this implementation), and $\theta_{m}$ the reflected angle at which the wavelength $\lambda$ has its maximum.

A second Newport 10D20ER.1 concave mirror placed in front of the holographic grating ensures that only one of the reflected wavelengths (the one travelling in the same direction as that given by $\theta_{m}$ ) will be directed to the detector. An RS Components 440-420 unipolar stepper motor ${ }^{6}$ changes the direction of the holographic grating relative to the second concave mirror, making it possible to select the wavelength directed to the detector. The position of the stepper motor is controlled by the control and processing unit, which also implements a microstepping technique based on pulse width modulation (PWM) in order to increase its angular resolution (which, in turn, leads to an improvement in the spectral resolution of $1 \mathrm{~nm}$ ). The reference position of the stepper motor is provided by a Fairchild Semiconductor H21A2 optocoupler. ${ }^{7}$ It is placed on the stepper motor and it triggers a signal to the control and processing unit whenever the stepper motor turns to this reference position. This reference position is used to calibrate the spectrometer each time the prototype is started up.

- Detector: the detector consists in a Thorlabs PDA-55 Si photodetector. ${ }^{8}$ It converts the optical beam coming from the wavelength separator into an electrical signal. Finally, the converted electrical signal is sent to the control and processing unit.

Figure 3 shows the spectral properties of the performance of the main optical components involved in the spectrometer. These characteristics will be taken into account by the "OSAgui" Windows application in order to compensate for the differences in the electrical output as a function of the detected wavelength.

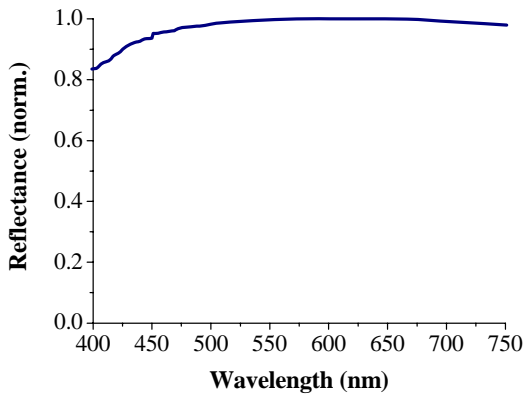

(a) Reflectance of the ER-1 Aluminum AR coating in the Newport 10D20 Metallic Mirror.

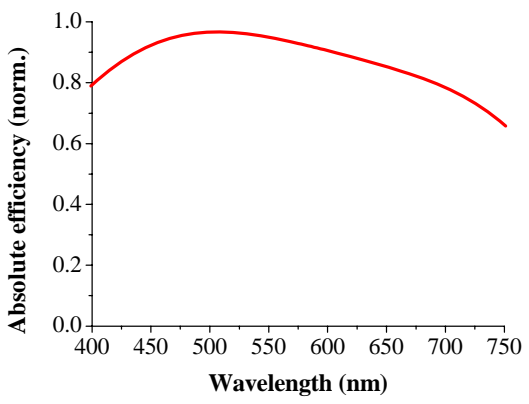

(b) Absolute efficiency of the holographic grating Edmund Optics NT43-225.

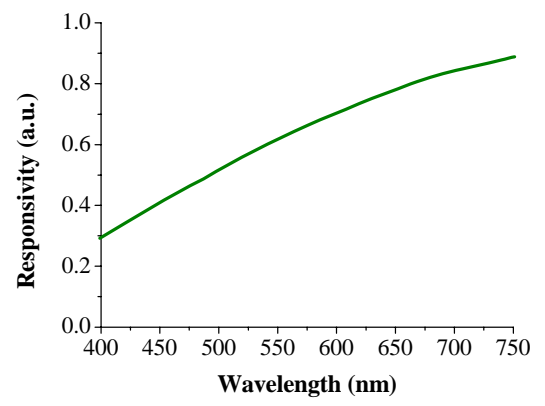

(c) Responsivity of the Thorlabs PDA55 Si photodetector.

Figure 3. Normalized reflectance, absolute efficiency, and responsivity of the concave mirrors, the reflective holographic grating, and the photodetector, respectively, against the wavelength.

\subsection{Control and processing unit}

The control and processing unit consists in a Digital Signal Processor (DSP), a TMS320F2812 model from Texas Instruments, ${ }^{9}$ and several dedicated signal conditioning circuits that adapt the different signals that 
enter or exit the DSP. Figures 4 and 5 show the block diagram and the photographs of the electronic circuits, as well as a general view of the final implementation.

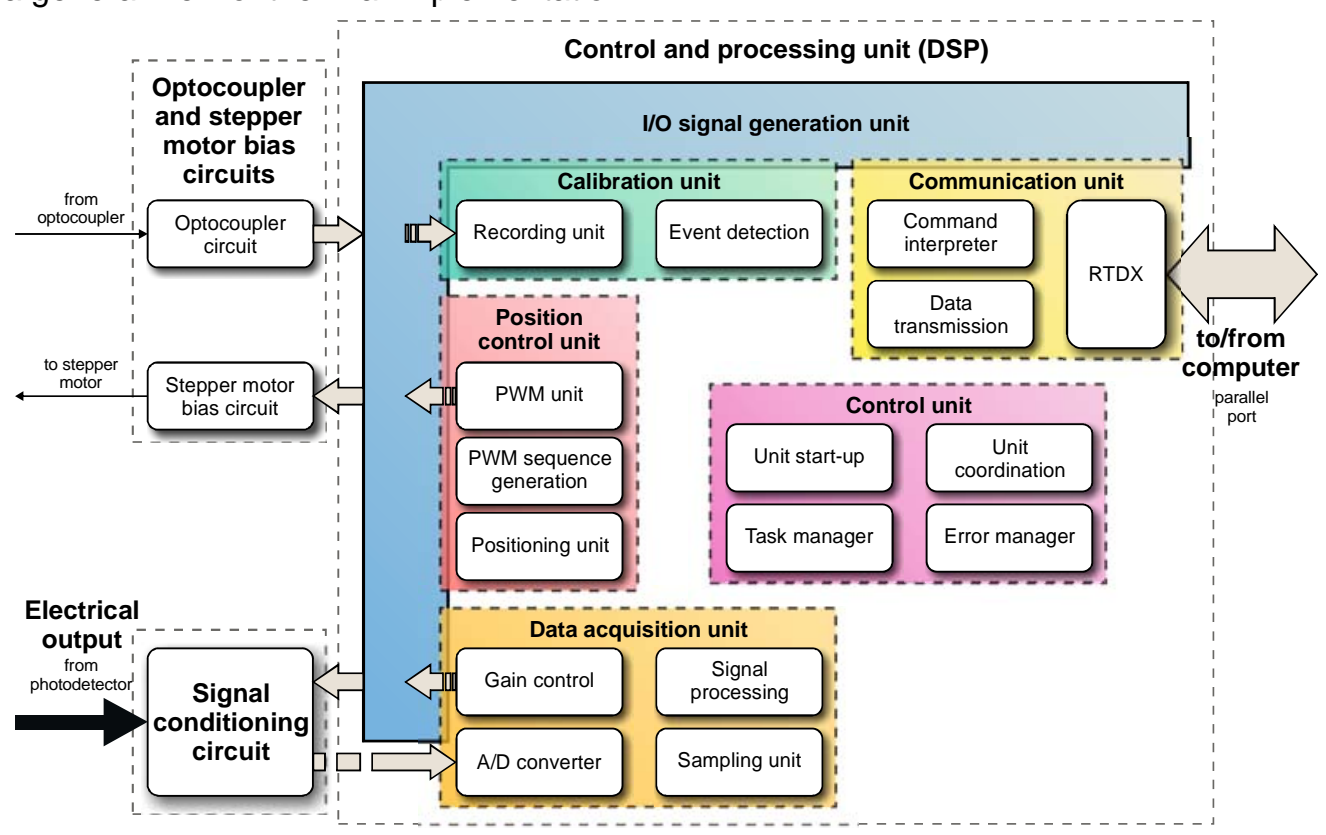

Figure 4. Block diagrams of the electronic circuits and of the control and processing unit.

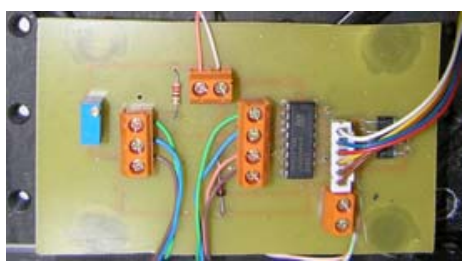

(a) Optocoupler and stepper motor bias circuits.

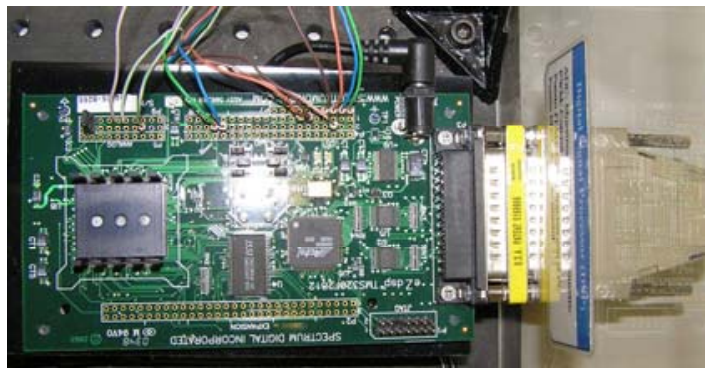

(c) Control and processing unit (DSP).

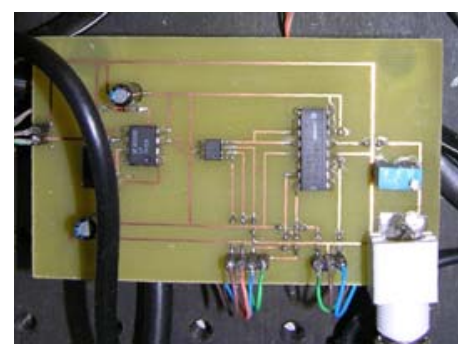

(b) Signal conditioning circuit.

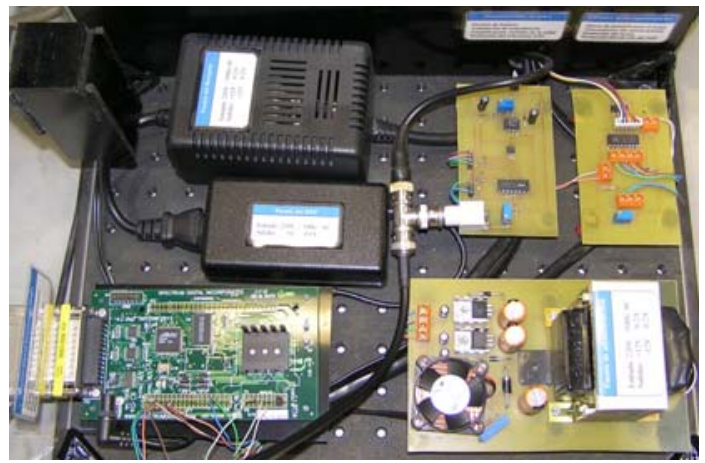

(d) General view. Both the electronic circuits and the control and processing unit are interspersed with several power supply units.

Figure 5. Photographs of the electronic circuits and of the control and processing unit. 
The DSP has been programmed using the Code Composer Studio, an integrated development environment provided by the manufacturer. The structure of the software (shown in Fig. 4) is described as follows:

- Data acquisition unit: it is responsible for digitalizing the electrical output of the spectrometer (previously conditioned by the signal conditioning circuit) and for processing this digitalized signal. Depending on the strength of the electrical output, the data acquisition unit sends the appropriate control signals (via $\mathrm{I} / \mathrm{O}$ signal generation unit) to the signal conditioning circuit to increase or reduce the gain accordingly.

- Communication unit: it links the DSP and the "OSAgui" Windows application installed on the PC together (the physical connection is made through the parallel port). It interprets the commands sent by the "OSAgui" Windows application (according to the requests made by the user) and sends the measurement results provided by the data acquisition unit to the "OSAgui" Windows application (so as to display them to the user).

- Position control unit: it is responsible for controlling the angular position of the stepper motor during the spectral sweep by sending the appropriate PWM signals.

- Calibration unit: it calibrates the spectrometer when the prototype is started up. For this purpose, the DSP changes sequentially the angular position of the stepper motor until the optocoupler triggers a signal; this angular position is assigned to the reference wavelength.

- Control unit: it controls and coordinates each one of the units, monitors the status of the DSP, and synchronizes in real time the different tasks that have to be carried out.

\section{3. "OSAgui" Windows application}

The "OSAgui" Windows application is the interface between the user and the control and processing unit: on the one hand, it gathers the requests made by the user and sends the corresponding commands to the control and processing unit; on the other hand, it receives the measurement results from the control and processing unit, performs a further processing, and displays the final results to the user. "OSAgui" is a userfriendly application and it has been developed in Visual Basic 6.0. Figure 6 shows its logical structure and a screenshot displaying the power spectrum of a white light emitting source.

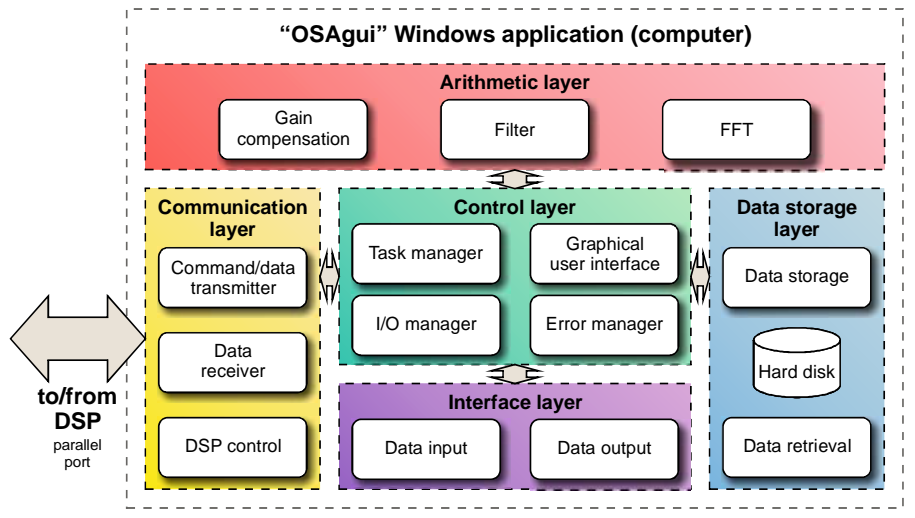

(a) Block diagram.

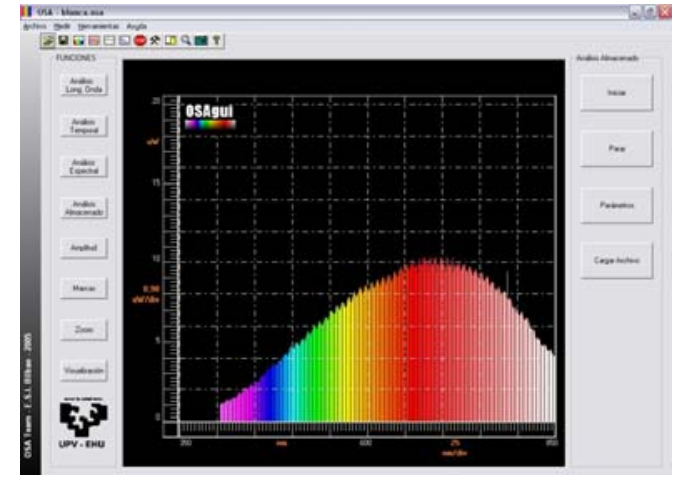

(b) Screenshot.

Figure 6. Block diagram and screenshot of the "OSAgui" Windows application.

"OSAgui" is structured as follows:

- Communication layer: it performs the required operations to establish, maintain and stop the bidirectional communication with the DSP. It sends the pertinent commands to the DSP and receives the data containing the measurement results from the DSP.

- Arithmetic layer: it processes the data provided by the communication layer. Its main task is to compensate for the variation in the obtained spectral power with the wavelength (see subsection 2.1 and Fig. 3 for further details). If requested by the user, it also filters the compensated measurement results and performs a Fast Fourier Transform. ${ }^{10}$

- Data storage layer: it stores the data provided by the arithmetic layer in the hard disk and vice versa. 
- Interface layer: it is the layer that interacts with the user. On the one hand, it prompts the user to enter the analysis parameters and other required data in the fields provided by the corresponding dialog box. On the other hand, it displays the obtained measurement results to the user.

- Control layer: it distributes the tasks to the corresponding layers and coordinates them, processes the data for its graphical representation by the interface layer, and monitors and handles every error and exception thrown during a certain operation.

\section{OPTICAL TIME DOMAIN REFLECTOMETER}

The designed OTDR prototype is suitable to characterize $9 / 125 \mu \mathrm{m}$ single-mode optical fibers operating in the second transmission window $(1310 \mathrm{~nm})$. This prototype has a maximum measuring range of $15 \mathrm{~km}$, a distance resolution of $20 \mathrm{~m}$, and an attenuation measurement resolution of $0.2 \mathrm{~dB}$. Figure 7 summarizes the four main components that constitute the OTDR prototype:

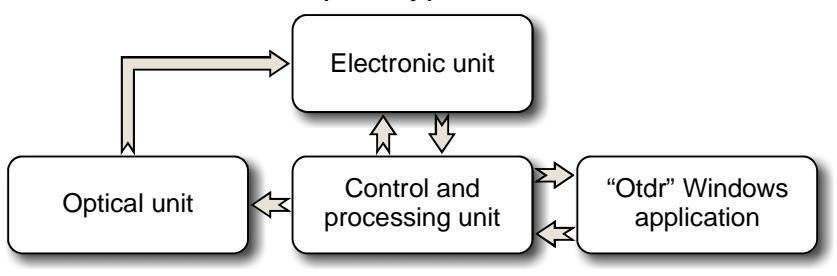

(a) Block diagram.

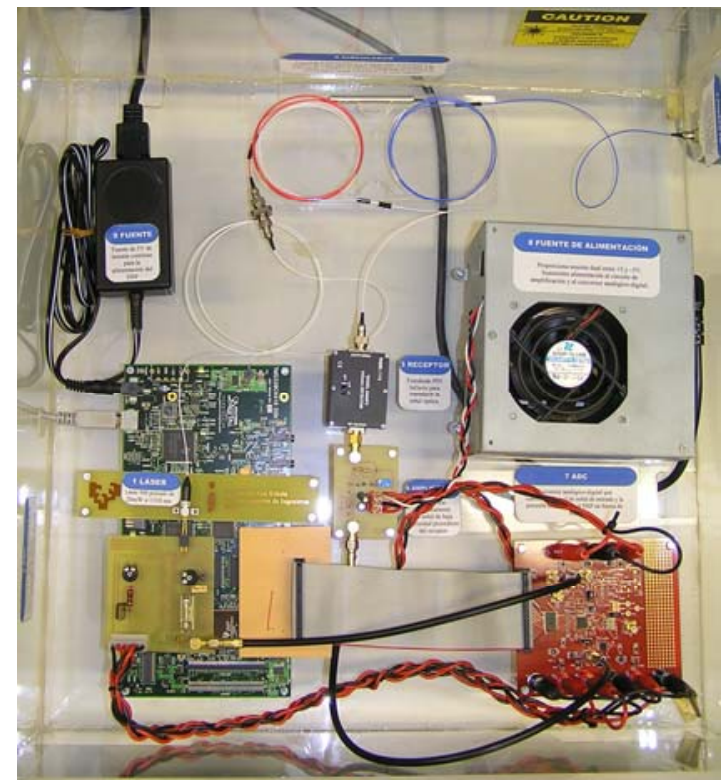

(b) Photograph of the components (interspersed with several power supply units) that constitute the prototype.

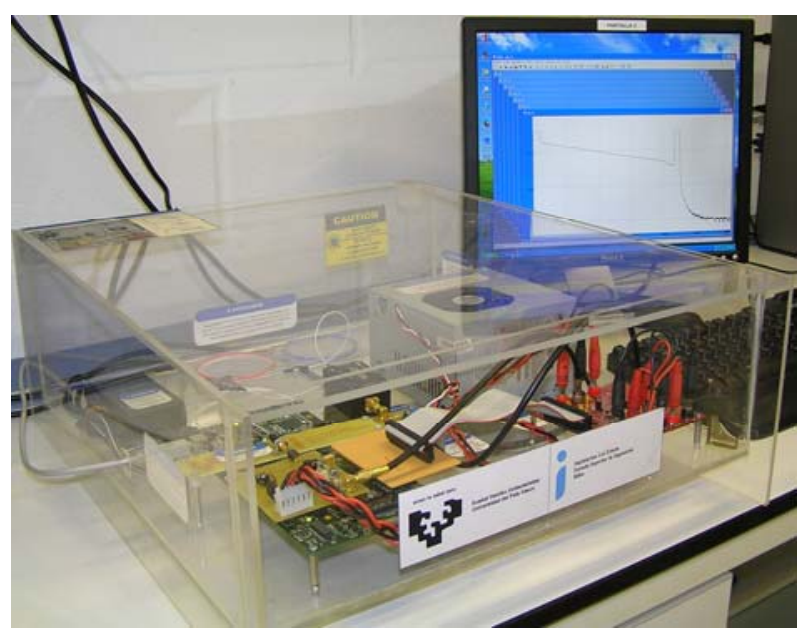

(c) Photograph of the prototype and the "Otdr" Windows application running on a PC.

Figure 7. Overall block diagram and two photographs of the OTDR prototype.

- Optical unit: the optical part that injects optical pulses into the fiber under test and converts the backscattered optical power from this fiber to an electrical signal. ${ }^{11}$

- Electronic unit: a set of devices and custom-made electrical circuits that adapt the electrical signal provided by the optical unit and sample it to a discrete signal.

- Control and processing unit: a general purpose DSP that controls the optical and electronic units. It processes the sampled data provided by the electronic unit.

- "Otdr" Windows application: a graphical user interface that manages the DSP and displays the obtained results. 


\subsection{Optical unit}

The main components of the optical unit are the laser diode, the optical circulator, and the photodetector. The block diagram of Fig. 8(a) shows the layout of the optical unit. Additionally, Figs. 8(b)-(d) show photographs of each component.

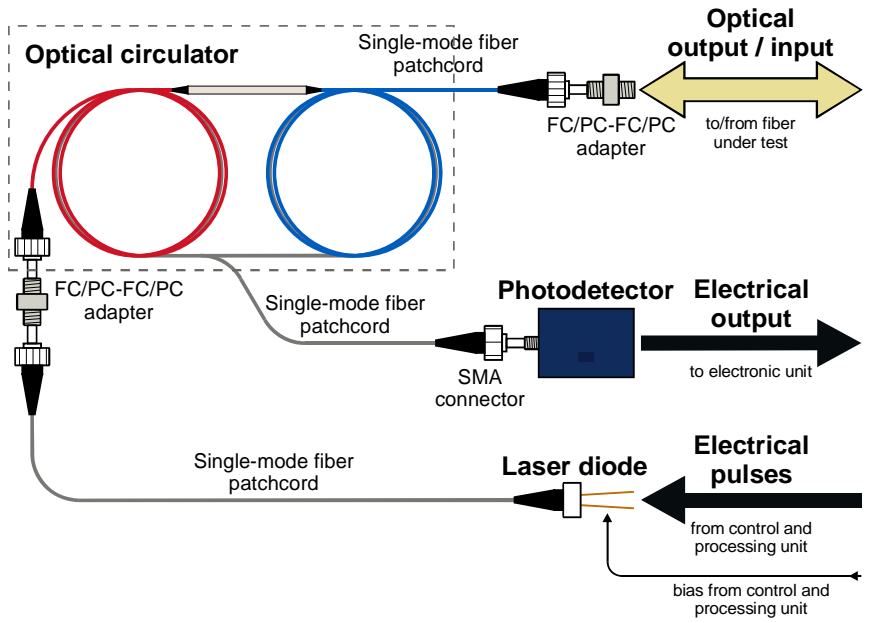

(a) Block diagram.

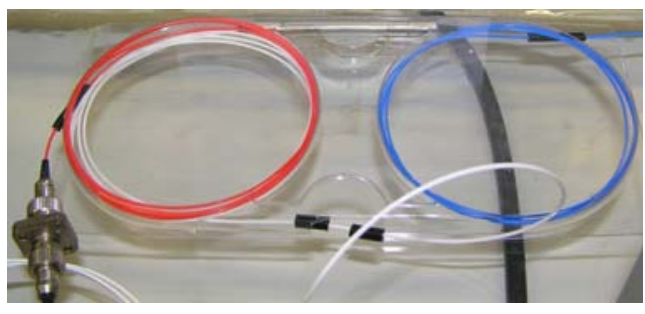

(b) Optical circulator

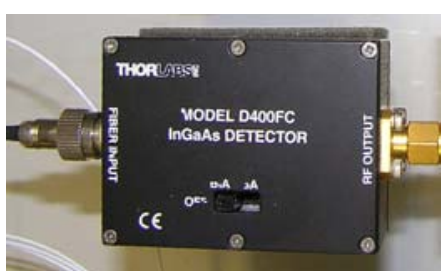

(c) Photodetector.

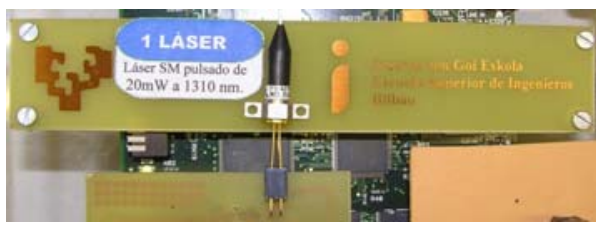

(d) Laser diode.

Figure 8. Block diagram and photographs of each component of the optical unit.

- Laser diode: it consists in a Mitsubishi FU-427SLD-F1M54 module that operates at $1310 \mathrm{~nm}^{12}$ The laser diode transforms the electrical pulses generated by the control and processing unit into optical pulses.

- Optical circulator: this is a polarization insensitive optical circulator from AC Photonics, which transfers the optical pulses from its first port to its second port (i.e., to the output of the OTDR) and the backscattered power from its second port to its third port (i.e., to the photodetector). ${ }^{13}$

- Photodetector: this is a very high speed Thorlabs D400FC InGaAs photodetector. ${ }^{14}$ It converts the backscattered power from the optical fiber under test into an electrical signal, which is sent to the electronic unit.

\subsection{Electronic unit}

The electronic unit consists of two main components (Fig. 9): 


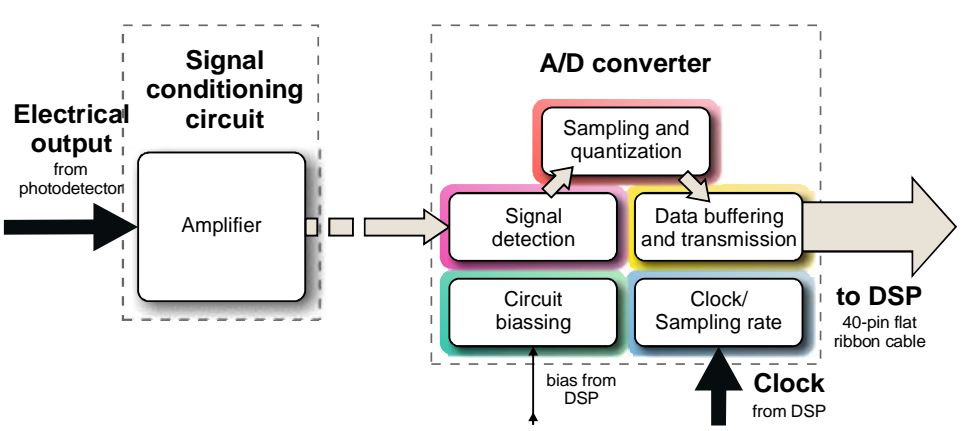

(a) Block diagram.

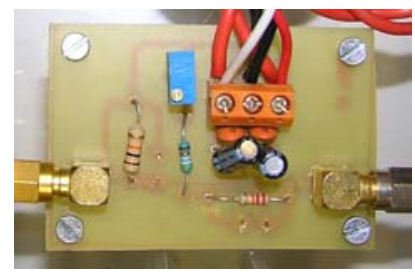

(b) Photograph of the signal conditioning circuit (the amplifier is placed on the back).

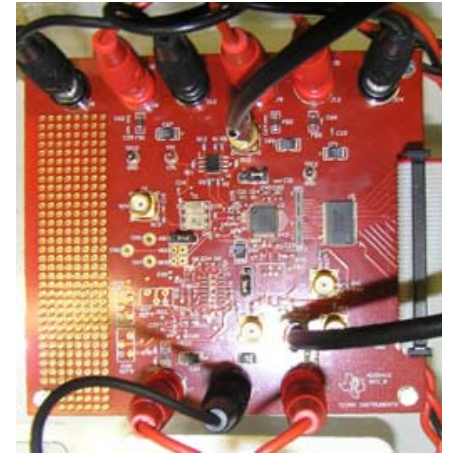

(c) Photograph of the A/D converter.

Figure 9. Block diagram and photographs of each component of the electronic unit.

- Signal conditioning circuit: this dedicated electronic circuit amplifies and makes suitable the electrical output from the photodetector before it is fed into the A/D converter. For such a purpose, it features a THS3001 high speed amplifier from Texas Instruments. ${ }^{15}$

- Analog-to-digital (A/D) converter: the main component is an ADS5410 integrated circuit, which is mounted on an ADS5410EVM evaluation module that provides all the necessary interconnection elements and sockets. ${ }^{16,17}$ The A/D converter samples the adapted electrical signal from the signal conditioning circuit and sends it to the control and processing unit via 40-pin flat ribbon cable. The sampling rate is obtained from the clock sent by the control and processing unit through a coaxial RF cable.

\subsection{Control and processing unit}

The control and processing unit is a Texas Instruments TMS320C6416 DSP. ${ }^{18}$ Figure 10 shows the logical structure of the software implemented in this DSP (using the Code Composer Studio environment):

- Data acquisition unit: it reads the digitalized data from the $A / D$ converter and processes them according to the parameters given by the configuration unit (this digitalized data represents the backscattered power as a function of the distance, from now on, the OTDR trace). Due to high speed requirements, the data acquisition unit makes use of the Enhanced Direct-Memory-Access built-in controller (via Memory Expansion Connector).

- Communication unit: it links the DSP and the "Otdr" Windows application installed on the PC together. Its purpose is to receive the commands sent by the "Otdr" Windows application and to send the OTDR traces back to the Windows application. The physical connection is made through the USB port.

- Laser diode pulse generation unit: it sends the signal pulses to the optical unit by means of the General-Purpose Input/Output peripheral (via Peripheral Expansion Connector). The duration of the pulses is set according to the parameters given by the configuration and control unit. 
- $A / D$ converter and laser diode biassing unit: it controls through the Peripheral Expansion Connector the biassing of the A/D converter and the laser diode.

- $\quad A / D$ converter control unit: it generates the clock signal and sends it to the A/D converter through the Peripheral Expansion Connector.

- Configuration and control unit: it initializes the DSP, interprets the commands provided by the communication unit, and configures and coordinates the memory, the external peripherals, and the rest of the units with the appropriate parameters in order to carry out the reflectometry measurements.

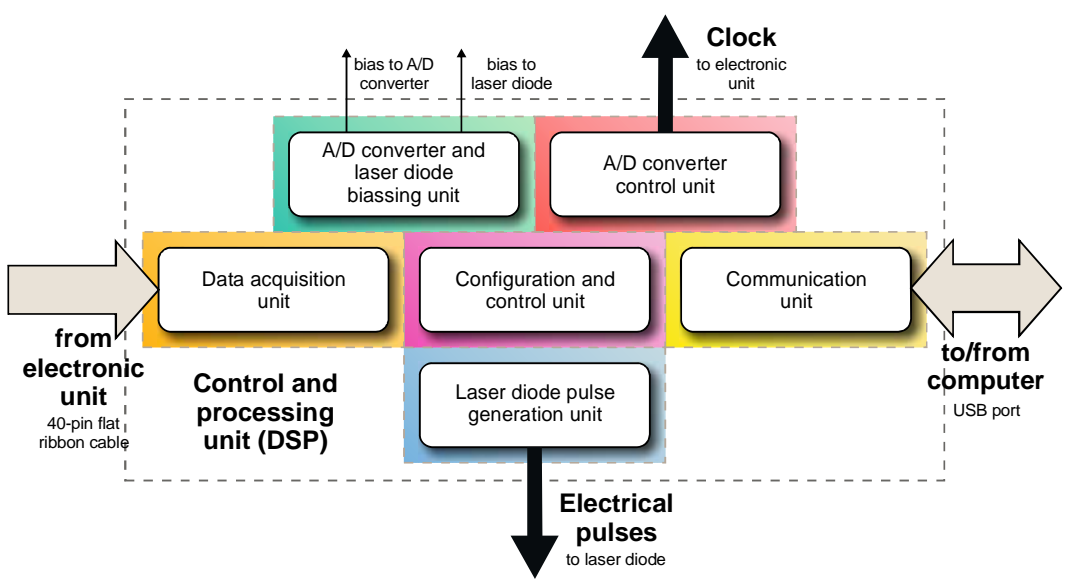

(a) Block diagram.

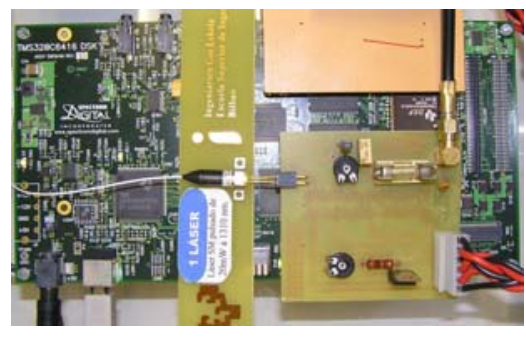

(b) Photograph.

Figure 10. Block diagram and photograph of the control and processing unit.

\section{4. "Otdr" Windows application}

The "Otdr" Windows application is the interface between the user and the control and processing unit. Its purpose is two-fold: to handle the requests from the user so as to send the appropriate instructions to the control and processing unit and to receive the measurement results from the latter in order to process them and display the OTDR trace, along with the detected events. The structure of this application, which has been developed in Visual C++ 6.0 using the Microsoft Foundation Class library, is shown in Fig. 11.

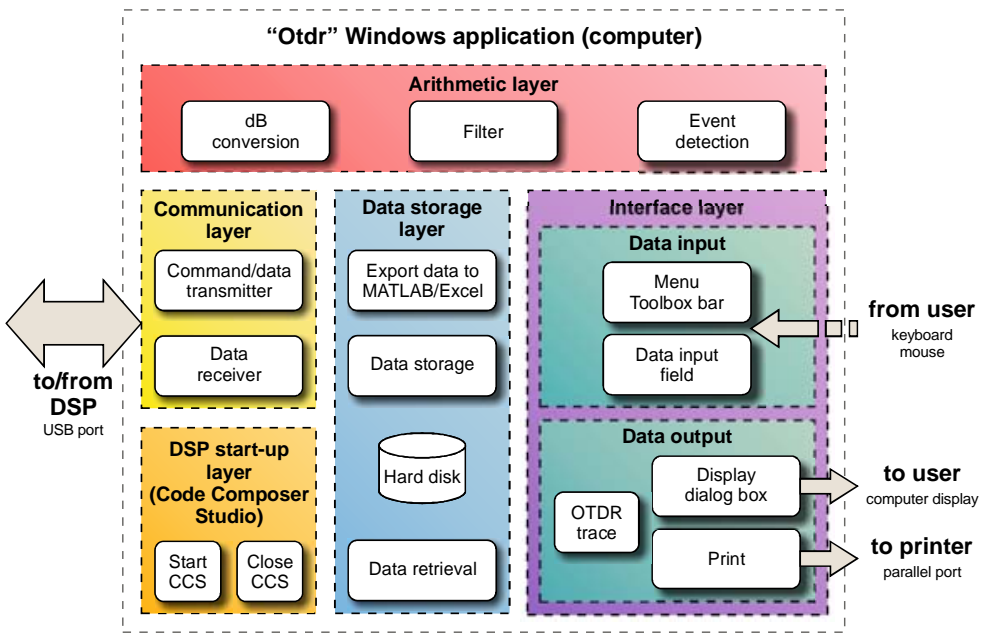

(a) Block diagram.

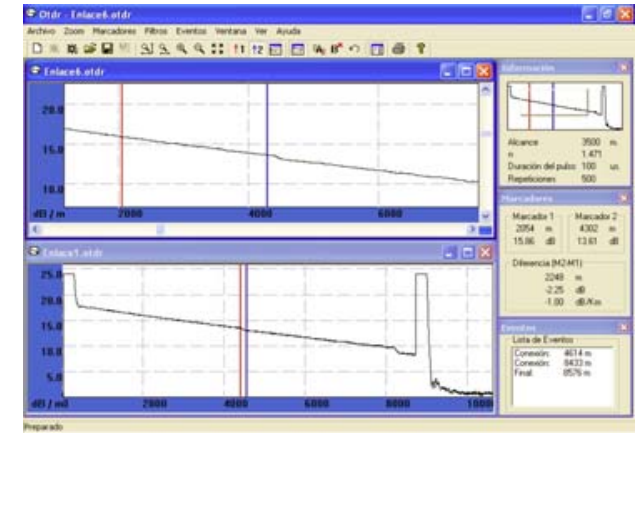

(b) Screenshot.

Figure 11. Block diagram and screenshot of the "Otdr" Windows application. 
- Communication layer: it is responsible for the bidirectional communication between the "Otdr" Windows application and the DSP. On the one hand, it sends the pertinent commands to the DSP to carry out the measurements; on the other hand, it receives the OTDR trace from the DSP.

- DSP start-up layer: this layer dumps the DSP routines to the RAM of the DSP each time the prototype is switched on. This is accomplished by running a script from the Code Composer Studio.

- Arithmetic layer: first of all, it converts the trace provided by the communication layer to logarithmic units (for efficiency reasons the DSP only handles linear data). Afterwards, it filters the trace in order to increase the signal-to-noise ratio and, finally, scans the trace for events. ${ }^{19}$

- Data storage layer: on the one hand, it stores/retrieves the trace in/from the hard disk; on the other hand, it performs the corresponding operations in order to export the measurement results to a spreadsheet, such as Microsoft Excel, or to MATLAB.

- Interface layer: it allows the user to enter the analysis parameters in a very intuitive way (using very simple dialog boxes, menus and toolboxes). It also displays the OTDR trace and information about the detected events (fiber losses, insertion losses, etc.) and it provides the user with tools such as markers that enable the analysis of single events.

In this application, each layer is responsible for the task it has been designed for. Accordingly, any error or exception is handled by the corresponding layer.

\section{CONCLUSIONS}

We have described in detail the design, structure, and implementation of two optical measurement systems, an OSA and an OTDR. The modular design of both prototypes allows a straightforward identification of the constituent parts, as well as a clear separation of the different tasks they are involved in. These features make both prototypes suitable for educational purposes and allow easy upgrade to include new functionalities or to improve existing capabilities.

\section{ACKNOWLEDGMENTS}

The authors would like to thank I. Blanco, I. Guereñu, R. Barreda, J. Roa, and J. M. Iglesias for their support and assistance with the development of the optical measurement systems.

This work was supported by the institutions Ministerio de Educación y Ciencia, Ministerio de Ciencia e Innovación, Universidad del País Vasco/Euskal Herriko Unibertsitatea, Gobierno Vasco/Eusko Jaurlaritza, Diputación Foral de Bizkaia/Bizkaiko Foru Aldundia, and the European Union 7th Research Framework Programme, under projects TEC2006-13273-C03-01, PSS-370000-2008-39, UE08/16, S-PE08CA01, DIPE08/24, and CE07/12-AISHA II, respectively.

\section{REFERENCES AND LINKS}

[1] Derickson D., "Fiber optic test and measurement," Prentice Hall, New Jersey, 1998.

[2] Thorlabs GmbH: "F230SMA-A Collimator," URL http://www.thorlabs.com/thorProduct.cfm?partNumber=F230SMA-A

[3] Newport Corporation: "10D20ER.1 Broadband Metallic Mirror," URL http://www.newport.com/Broadband-Metallic-Mirrors/141088/1033/catalog.aspx

[4] Edmund Optics Inc.: "NT43-225 Reflective Holographic Grating," URL http://www.edmundoptics.com/onlinecatalog/DisplayProduct.cfm?productid=1621

[5] Hecht E., "Optics," Addison Wesley, 4th ed., New York, 2002.

[6] RS Components Ltd.: "440-420 stepper motor," URL http://uk.rsonline.com/web/search/searchBrowseAction. html?method=getProduct\&R=0440420

[7] Fairchild Semiconductor Inc.: "H21A2 Slotted Optical Switch," URL http://www.fairchildsemi.com/pf/H2/H21A2.html

[8] Thorlabs GmbH: "PDA55 Amplified Si Photodetector," URL http://www.thorlabs.com/thorProduct.cfm?partNumber=PDA55

[9] Texas Instruments Inc.: "TMS320F2812 Digital Signal Controller," URL http://focus.ti.com/docs/prod/folders/print/tms320f2812.html 
[10] Proakis J. G. and Manolakis D. G., "Digital Signal Processing. Principles, algorithms, and applications," Prentice Hall, 3rd ed., London, 1997.

[11] Kapron F. P., Adams B. P., Thomas E. A., and Peters J.W, "Fiber-optic reflection measurements using OCWR and OTDR techniques," IEEE Journal of Lightwave Technology, vol. 7, no. 8, pp. 1234 - 1241, Aug 1989.

[12] Mitsubishi Electric Corp.: "FU-427SLD-F1M54 Laser Diode," URL http://www.mitsubishichips.com/Global/products/opt/index.html

[13] AC Photonics Inc.: "Polarization insensitive optical circulator," URL http://www.acphotonics.com/products/product files/Polarization\%20Insensitive\%200ptical\%20Circulat or.htm

[14] Thorlabs GmbH: "D400FC InGaAs Photodetector," URL http://www.thorlabs.com/thorProduct.cfm?partNumber=D400FC

[15] Texas Instruments Inc.: "THS3001 Current-Feedback Amplifier," URL http://focus.ti.com/docs/prod/folders/print/ths3001.html

[16] Texas Instruments Inc.: "ADS5410 Analog-to-Digital Converter," URL http://focus.ti.com/docs/prod/folders/print/ads5410.html

[17] Texas Instruments Inc.: "ADS5410EVM Evaluation Module," URL http://focus.ti.com/docs/toolsw/folders/print/ads5410evm.html

[18] Texas Instruments Inc.: "TMS320C6416 Fixed-Point Digital Signal Processor," URL http://focus.ti.com/docs/prod/folders/print/tms320c6416.html

[19] Press W. H., Flannery B. P., Teukolsky S. A., and Vetterling W. T., "Numerical recipes in C: The art of scientific computing," Cambridge University Press, Cambridge, 2002. 